\title{
Fault-Tolerant Control for Networked Control Systems with Limited Information in Case of Actuator Fault
}

\author{
Wang Yan-feng, Wang Pei-liang, Li Zu-xin, and Chen Hui-ying \\ School of Engineering, Huzhou University, Huzhou, Zhejiang 313000, China \\ Correspondence should be addressed to Wang Yan-feng; neu2009wyf@163.com
}

Received 3 January 2015; Revised 17 February 2015; Accepted 25 February 2015

Academic Editor: Hui Zhang

Copyright (C) 2015 Wang Yan-feng et al. This is an open access article distributed under the Creative Commons Attribution License, which permits unrestricted use, distribution, and reproduction in any medium, provided the original work is properly cited.

This paper is concerned with the problem of designing a fault-tolerant controller for uncertain discrete-time networked control systems against actuator possible fault. The step difference between the running step $k$ and the time stamp of the used plant state is modeled as a finite state Markov chain of which the transition probabilities matrix information is limited. By introducing actuator fault indicator matrix, the closed-loop system model is obtained by means of state augmentation technique. The sufficient conditions on the stochastic stability of the closed-loop system are given and the fault-tolerant controller is designed by solving a linear matrix inequality. A numerical example is presented to illustrate the effectiveness of the proposed method.

\section{Introduction}

Networked control systems (NCSs) are used in many fields such as remote surgery and unmanned aerial vehicles especially in a number of emerging engineering applications such as arrays of microactuators and even neurobiological and socialeconomical systems [1-3]. Compared with the traditional wiring, the communication channels can simplify the installation and reduce the costs of cables and maintenance of the system. However, the network in the control systems also brings many problems, such as network-induced delay and packet dropout, and makes system analysis more challenging $[4,5]$. Network-induced delays can degrade the performance of control systems designed without considering them and even destabilize the system $[6,7]$.

Because of the complexity caused by network, NCSs are more vulnerable to faults. An effective way to increase the reliability of the NCSs is to introduce fault-tolerant control (FTC). Therefore, the research on fault-tolerant control of NCSs has great theoretical and applied significance; however research on FTC for NCSs is different from that for traditional control systems in many aspects $[8,9]$. In [10], a fault estimator was proposed for NCSs with transfer delays, process noise, and model uncertainty. On the basis of the information on fault estimator, a fault-tolerant controller using sliding mode control theory was designed to recover the system performance. In [11], the random packet dropout and the sensor or actuator failure were described as binary random variables; the sufficient condition for asymptotical meansquare stability of the NCSs was derived. By using matrix measure technique, a fault-tolerant controller was designed for NCSs with network-induced delay and model uncertainty in [12]. In [13], a FTC algorithm considering actuator failure of an NCS was presented, and the NCS with data packet dropout was modeled as an asynchronous dynamical system. Based on information scheduling, FTC design methods were proposed for NCSs with communication constrains in [14]. In [15], the problem of fault-tolerant control for NCSs with data packet dropout is studied and the closed-loop system was modeled as Markov jump system. However, elements of transition probabilities matrix are assumed to be completely known and the controller can not be solved by LMIs. To the best of the authors' knowledge, up to now, very limited efforts have been devoted to studying FTC for uncertain NCSs with uncertain transition probability matrices, which motivates our investigation.

Problems of partial sensors inactivation are equal to problems of data pack dropout which can be solved by common technique; we focus on the problems of reliability when actuators are inactivated in this paper. 
In this paper, the step difference $\tau_{k}$ between the running step $k$ and the time stamp of the used plant state is modeled as a finite state Markov chain. And the information of the transition probabilities matrix is limited; that is, a part of elements of transition probabilities matrix is unknown. The closed-loop system model is obtained by means of state augmentation technique and the mode-dependent faulttolerant controller is designed which guarantees the stochastic stability of the closed-loop system.

This paper is organized as follows. In Section 2, we formulate the state feedback controller design problem. In Section 3, the sufficient conditions to guarantee the stochastic stability are presented, and the fault-tolerant controller is also given. A simulation example is used to illustrate the effectiveness of the proposed method in Section 4 . The conclusion remarks are addressed in Section 5.

\section{Problem Formulation}

Consider the NCSs setup in Figure 1, in which the controllers are placed in a remote location, and both sensor measurement data and control data are transmitted through network.

By adding a buffer to the actuator, the delay $\tau_{k}^{\text {sc }}$ from sensor to controller and the delay $\tau_{k}^{\mathrm{ca}}$ from controller to actuator can be lumped together, and the new variable is described as $\tau_{k}=\tau_{k}^{\mathrm{sc}}+\tau_{k}^{\mathrm{ca}}$ which is modeled as a Markov chain. And $\tau_{k}$ denotes the step difference between the running step $k$ and the time stamp of the used plant state, and it depends on the random time-delay and the data packet drops on the random communication delay and the data packet dropout [16]. Assume that both time-delay and the data packet dropout are bounded, so $\tau_{k}$ is bounded. The step delay $\tau_{k}$ takes values in $\Lambda=\{1,2, \ldots, d\}$ and the transition probability matrix of $\tau_{k}$ is $\Pi=\left[\pi_{i j}\right]$. That is, $\tau_{k}$ jumps from mode $i$ to $j$ with probability $\pi_{i j}$ which is defined by $\pi_{i j}=\operatorname{Pr}\left(\tau_{k+1}=j \mid \tau_{k}=i\right)$, where $\pi_{i j} \geq 0, \forall i, j \in \Lambda$, $\sum_{j=1}^{d} \pi_{i j}=1$. The set $\Lambda$ contains $d$ modes of $\tau_{k}$, and the transition probabilities of the jumping process in this paper are considered to be partly accessed; that is, some elements in matrix $\Pi$ are unknown. For example, for the time-delay $\tau_{k}$ with 3 modes, the transition probabilities matrix $\Pi$ may be as follows:

$$
\Pi=\left[\begin{array}{ccc}
? & \pi_{12} & ? \\
\pi_{21} & ? & ? \\
? & \pi_{32} & ?
\end{array}\right]
$$

where "?" represents the inaccessible elements. For notational clarity, $\forall i \in \Lambda$, we denote $\Lambda=\Lambda_{k}^{i}+\Lambda_{u k}^{i}$ with

$$
\begin{gathered}
\Lambda_{k}^{i}=\left\{j: \pi_{i j} \text { is known }\right\}, \\
\Lambda_{u k}^{i}=\left\{j: \pi_{i j} \text { is unknown }\right\} .
\end{gathered}
$$

Moreover, if $\Lambda_{k}^{i} \neq \emptyset$, it is further described as $\Lambda_{k}^{i}=\left\{k_{1}^{i}, k_{2}^{i}\right.$, $\left.\ldots, k_{\mu}^{i}\right\}, 1 \leq \mu \leq d$, where $k_{\mu}^{i}$ represents the $\mu$ th known element with the index $k_{\mu}^{i}$ in the $i$ th row of the matrix $\Pi$.

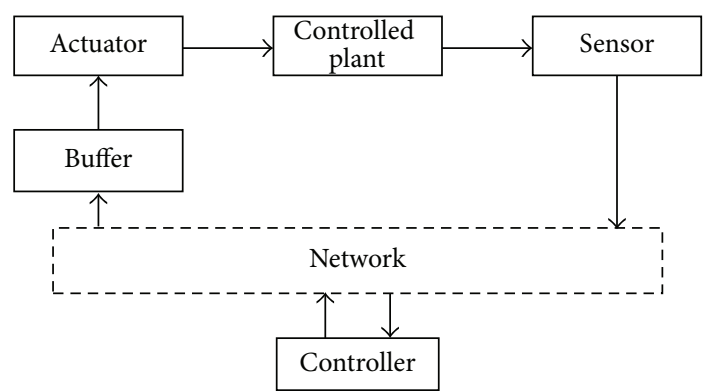

FIGURE 1: Structure of networked control system.

And $\Lambda_{u k}^{i}$ is described as $\Lambda_{u k}^{i}=\left\{\bar{k}_{1}^{i}, \bar{k}_{2}^{i}, \ldots, \bar{k}_{d-\mu}^{i}\right\}$, where $\bar{k}_{d-\mu}^{i}$ represents the $(d-\mu)$ th unknown element with the index $(d-\mu)$ th in the $i$ th row of the matrix $\Pi$.

Assume that the model of the plant is an uncertain discrete-time system as follows:

$$
x(k+1)=\left(A_{0}+\Delta A_{0}\right) x(k)+\left(B_{0}+\Delta B_{0}\right) u(k),
$$

where $x(t) \in R^{n}$ is state vector and $u(t) \in R^{m}$ is the control input. $A_{0} \in R^{n \times n}$ and $B_{0} \in R^{n \times m}$ are all real constant matrices. $\left[\begin{array}{cc}\Delta A_{0} & \Delta B_{0}\end{array}\right]=D F(k)\left[\begin{array}{ll}E_{1} & E_{2}\end{array}\right]$, where $F(k)$ is an uncertain time-varying matrix satisfying the bound $F(k)^{T} F(k)<I$, where $I$ denotes the identity matrix with appropriate dimension.

Considering the effect of the random communication delay and the data packet dropout, we describe the state feedback control law as

$$
\begin{gathered}
u(k)=K\left(\tau_{k}\right) x\left(k-\tau_{k}\right), \\
x(0)=x_{0} \in R^{n} .
\end{gathered}
$$

The fault indicator matrix $L$ is given by

$$
L=\operatorname{diag}\left\{l_{1}, \ldots, l_{m}\right\}
$$

with $l_{j} \in\{0,1\}$ for $j=1,2, \ldots, m$ and $l_{j}=0$ means the $j$ th actuator experiences a total failure, whereas the $j$ th actuator is in healthy state when $l_{j}=1$. Since there are $m$ actuators, the set of possible related failure modes is finite and is denoted by $\widetilde{L}=\left\{L^{1}, \ldots, L^{N}\right\}$ with $2^{m}-1$ elements, where $L^{i}(i=$ $1,2, \ldots, N)$ is a particular pattern of matrix $L$.

Consequently, the closed-loop system from (3) and (4) can be expressed as

$$
\begin{aligned}
x(k+1)= & \left(A_{0}+\Delta A_{0}\right) x(k) \\
& +\left(B_{0}+\Delta B_{0}\right) L K\left(\tau_{k}\right) x\left(k-\tau_{k}\right) .
\end{aligned}
$$

At sampling time $k$, if we augment the state-variable as $\delta(k)=$ $\left[\begin{array}{llll}x(k)^{T} & x(k-1)^{T} & \cdots & x(k-d)^{T}\end{array}\right]^{T}$, the closed-loop system (6) can be written as

$$
\begin{aligned}
& \delta(k+1)=\left(A+B L K\left(\tau_{k}\right) H\left(\tau_{k}\right)\right) \delta(k), \\
& \delta(0)=\left[\begin{array}{llll}
\delta(0)^{T} & \delta(-1)^{T} & \cdots & \delta(-d)^{T}
\end{array}\right]^{T},
\end{aligned}
$$


where

$$
\begin{gathered}
A=\left[\begin{array}{ccccc}
A_{0}+\Delta A_{0} & 0 & \cdots & 0 & 0 \\
I & 0 & \cdots & 0 & 0 \\
0 & I & \cdots & 0 & 0 \\
\vdots & \vdots & \ddots & 0 & 0 \\
0 & 0 & \cdots & I & 0
\end{array}\right] \in R^{n(1+d) \times n(1+d)}, \\
B=\left[\begin{array}{ccc}
B_{0}+\Delta B_{0} \\
0 \\
0 \\
0 \\
\vdots \\
0
\end{array}\right] \in R^{n(2+1) \times m}, \\
H\left(\tau_{k}\right)=\left[\begin{array}{lllll}
0 & 0 & I & \cdots & 0
\end{array}\right] \in R^{n \times n(1+d)}
\end{gathered}
$$

has all elements being zeros except for the $\left(1+\tau_{k}\right)$ th block being identity.

It can be seen that the closed-loop system (7) is a jump linear system with $d$ different modes.

It is noticed that $A=\widetilde{A}_{0}+\Delta \widetilde{A}_{0}=\widetilde{A}_{0}+\widetilde{D F} \widetilde{E}_{1}$ and $B=$ $\widetilde{B}_{0}+\Delta \widetilde{B}_{0}=\widetilde{B}_{0}+\widetilde{D} F E_{2}$ where

$$
\begin{gathered}
\widetilde{A}_{0}=\left[\begin{array}{ccccc}
A_{0} & 0 & \cdots & 0 & 0 \\
I & 0 & \cdots & 0 & 0 \\
0 & I & \cdots & 0 & 0 \\
\vdots & \vdots & \ddots & 0 & 0 \\
0 & 0 & \cdots & I & 0
\end{array}\right], \quad \Delta \widetilde{A}_{0}=\left[\begin{array}{ccccc}
D F E_{1} & 0 & \cdots & 0 & 0 \\
0 & 0 & \cdots & 0 & 0 \\
0 & 0 & \cdots & 0 & 0 \\
\vdots & \vdots & \ddots & 0 & 0 \\
0 & 0 & \cdots & 0 & 0
\end{array}\right], \\
\widetilde{D}=\left[\begin{array}{c}
D \\
0 \\
0 \\
\vdots \\
0
\end{array}\right], \quad \widetilde{B}_{0}=\left[\begin{array}{c}
B_{0} \\
0 \\
0 \\
\vdots \\
0
\end{array}\right], \Delta \widetilde{B}_{0}=\left[\begin{array}{c}
D F E_{2} \\
0 \\
0 \\
\vdots \\
0
\end{array}\right],
\end{gathered}
$$

Throughout this paper, we use the following definition.

Definition 1. System (7) is stochastically stable if for every finite $\delta(0)$ and initial mode $\tau_{0} \in \Lambda$ there exists a finite $W>0$ such that the following holds:

$$
E\left\{\sum_{k=0}^{\infty}\|\delta(k)\|^{2} \mid \delta_{0}, \tau_{0}\right\}<\delta_{0}^{T} W \delta_{0} .
$$

The object of this paper is to construct a fault-tolerant controller with structure as given by (4) which achieves that the closed-loop system (7) is stochastically stable under all actuator failure modes. In the following, $K\left(\tau_{k}\right)$ and $H\left(\tau_{k}\right)$ for this paper are denoted as $K(i)$ and $E(i)$, respectively.

To proceed, we will need the following two lemmas.

Lemma 2 (see [17]). Given matrices $W, M$, and $N$ of appropriate dimensions and $W$ is symmetric, $W+N^{T} F(k)^{T} M^{T}+$ $M F(k) N<0$ holds for all $F(k)$ satisfying $F(k)^{T} F(k) \leq I$ if and only if there exists a scalar $\varepsilon>0$ such that $W+\varepsilon M M^{T}+$ $\varepsilon^{-1} N^{T} N<0$.

Lemma 3 (see [18]). The matrix $E(i)^{T}$ is of full-array rank; then there exist two orthogonal matrices $U_{i} \in R^{n(1+d) \times n(1+d)}$ and $V_{i} \in R^{g \times g}$, such that $E(i)^{T}=U_{i}^{T}\left[\begin{array}{c}\Sigma_{i} \\ 0\end{array}\right] V_{i}^{T}$, where $\Sigma_{i}=$ $\operatorname{diag}\left(\sigma_{1}, \sigma_{2}, \ldots \sigma_{g}\right)$, where $\sigma_{i}(i=1,2, \ldots, g)$ are nonzero singular values of $E(i)^{T}$. If matrix $Q(i) \in R^{n(1+d) \times n(1+d)}$ has the following structure

$$
Q(i)=U_{i}^{T}\left[\begin{array}{cc}
Q_{i 1} & 0 \\
0 & Q_{i 2}
\end{array}\right] U_{i},
$$

there exists a nonsingular matrix $X_{i} \in R^{n \times n}$ such that $Q(i) E(i)^{T}=E(i)^{T} X_{i}$, where $Q_{i 1} \in R^{g \times g}>0, Q_{i 2} \in$ $R^{n(1+d-g) \times n(1+d-g)}>0$.

\section{Controller Design}

With Definition 1, the sufficient conditions on the stochastic stability of the closed-loop system (7) can be obtained.

Theorem 4. The closed-loop system (7) with partly unknown transition probabilities (2) is stochastically stable if there exists matrix $P(i)>0, i \in \Lambda$ such that

$$
\begin{aligned}
& A(i)^{T} \sum_{j \in \Lambda_{k}^{i}} \pi_{i j} P(j) A(i)-\sum_{j \in \Lambda_{k}^{i}} \pi_{i j} P(i)<0, \\
& A(i)^{T} P(j) A(i)-P(i)<0, \quad \forall j \in \Lambda_{u k}^{i},
\end{aligned}
$$

where $A(i)=A+B L K(i) H(i)$

Proof. For the closed-loop system (7), consider the quadratic function which is given by

$$
V(\delta(k), k)=\delta^{T}(k) P\left(\tau_{k}\right) \delta(k) .
$$

Then,

$$
\begin{aligned}
E\{\Delta & V(\delta(k), k)\} \\
= & E\left\{\delta^{T}(k+1) P\left(\tau_{k+1}\right) \delta(k+1) \mid \delta(k), \tau_{k}=i\right\} \\
& -\delta^{T}(k) P\left(\tau_{k}\right) \delta(k) \\
= & \delta(k)^{T}(A+B L K(i) H(i))^{T} \\
& \cdot \sum_{j=1}^{d} \pi_{i j} P(j)(A+B L K(i) H(i)) \\
& -\delta^{T}(k) P(i) \delta(k)
\end{aligned}
$$




$$
\begin{aligned}
= & \delta(k)^{T}\left(A(i)^{T} \sum_{j \in \Lambda_{k}^{i}} \pi_{i j} P(j) A(i)-\sum_{j \in \Lambda_{k}^{i}} \pi_{i j} P(i)\right) \\
& \cdot \delta(k)+\delta(k)^{T} \\
& \cdot\left(A(i)^{T} \sum_{j \in \Lambda_{u k}^{i}} \pi_{i j} P(j) A(i)-\sum_{j \in \Lambda_{u k}^{i}} \pi_{i j} P(i)\right) \delta(k) \\
= & \delta(k)^{T}\left(A(i)^{T} \sum_{j \in \Lambda_{k}^{i}} \pi_{i j} P(j) A(i)-\sum_{j \in \Lambda_{k}^{i}} \pi_{i j} P(i)\right) \delta(k) \\
& +\delta(k)^{T}\left(\sum_{j \in \Lambda_{u k}^{i}} \pi_{i j} A(i)^{T} P(j) A(i)-P(i)\right) \delta(k) .
\end{aligned}
$$

Hence, if (12) and (13) hold, $\Delta V(\delta(k), k)<0$. One has

$$
\begin{aligned}
E\{V(\delta(k), k)-V(\delta(0), 0)\} \\
=E\left\{\sum_{k=0}^{k} \Delta V(k)\right\} \leq \beta E\left\{\sum_{k=0}^{k}\|\delta(k)\|^{2}\right\},
\end{aligned}
$$

where $\beta=\lambda_{\min }(A+B L K(i) H(i))^{T} \sum_{j=1}^{d} \pi_{i j} P(j)(A+$ $B L K(i) H(i))-P(i)$; hence one can get $\lim _{k \rightarrow \infty} E\left\{\sum_{k=0}^{k}\right.$ $\left.\|\delta(k)\|^{2}\right\} \leq(1 / \beta) E\{V(0)\}$. According to Definition 1, system (7) is stochastically stable.

Clearly, no knowledge on $\pi_{i j}, \forall j \in \Lambda_{u k}^{i}$ is needed in (12) and (13), which completes the proof.

Theorem 5. Consider system (7) with partly unknown transition probabilities (2). If there exist matrices $Q(i)>0, Y(i)$ and positive scalars $\mu_{1}, \mu_{2}, \mu_{3}$, and $\mu_{4}$ such that

$$
\begin{aligned}
& {\left[\begin{array}{cccc}
-\sum_{j \in \Lambda_{k}^{i}} \pi_{i j} Q(i) & * & * & * \\
\Lambda_{k}^{i}\left(\widetilde{A}_{0}+\widetilde{B}_{0} L Y(i) H(i)\right) & \Gamma+\Xi_{k}^{i} & * & * \\
E_{2} L Y(i) H(i) & 0 & -\mu_{1} I & * \\
\widetilde{E}_{1} & 0 & 0 & -\mu_{2} I
\end{array}\right]<0,} \\
& {\left[\begin{array}{cccc}
-Q(i) & * & * & * \\
\widetilde{A}_{0}+\widetilde{B}_{0} L Y(i) H(i) & -Q(j)+\left(\mu_{3}+\mu_{4}\right) \widetilde{D} \widetilde{D}^{T} & * & * \\
E_{2} L Y(i) H(i) & 0 & -\mu_{3} I & * \\
\widetilde{E}_{1} Q(i) & 0 & 0 & -\mu_{4} I
\end{array}\right]} \\
& <0, \quad \forall j \in \Lambda_{u k}^{i} \text {, }
\end{aligned}
$$

where

$$
\begin{aligned}
& \Lambda_{k}^{i}=\left[\begin{array}{llll}
\sqrt{\pi_{i k_{1}^{i}}} I & \sqrt{\pi_{i k_{2}^{i}}} I & \cdots & \sqrt{\pi_{i k_{\mu}^{i}}} I
\end{array}\right]^{T}, \\
& \Xi_{k}^{i}=-\operatorname{diag}\left\{Q\left(k_{1}^{i}\right) Q\left(k_{2}^{i}\right) \cdots Q\left(k_{\mu}^{i}\right)\right\}, \\
& \Gamma=\left[\begin{array}{cccc}
\left(\mu_{1}+\mu_{2}\right) \widetilde{D} \widetilde{D}^{T} & * & * & * \\
\left(\mu_{1}+\mu_{2}\right) \widetilde{D} \widetilde{D}^{T} & \left(\mu_{1}+\mu_{2}\right) \widetilde{D} \widetilde{D}^{T} & * & * \\
\vdots & \vdots & \ddots & * \\
\left(\mu_{1}+\mu_{2}\right) \widetilde{D} \widetilde{D}^{T} & \left(\mu_{1}+\mu_{2}\right) \widetilde{D} \widetilde{D}^{T} & \cdots & \left(\mu_{1}+\mu_{2}\right) \widetilde{D} \widetilde{D}^{T}
\end{array}\right]
\end{aligned}
$$

then there exists a mode-dependent controller of the form (4) such that the resulting system (7) is stochastically stable. Furthermore, an admissible controller is given by

$$
K(i)=Y(i) V_{i} \Sigma_{i}^{-1} Q_{i 1}^{-1} \Sigma_{i} V_{i}^{T} .
$$

Proof. According to Theorem 4, we know that the system (7) is stochastically stable with the partly unknown transition probabilities (2) if inequalities (12) and (13) hold. By Schur complement, inequality (12) is equivalent to

$$
\left[\begin{array}{cc}
-\sum_{j \in \Lambda_{k}^{i}} \pi_{i j} P(i) & * \\
\Lambda_{k}^{i}\left(A+\widetilde{B}_{0} L K(i) H(i)\right) & \Omega_{k}^{i}
\end{array}\right]+\Psi F \Theta+\Psi^{T} F^{T} \Theta^{T}<0,
$$

where $\Psi=\left[\begin{array}{lllll}0 & \widetilde{D}^{T} & \ldots & \widetilde{D}^{T} & \widetilde{D}^{T}\end{array}\right]^{T}, \quad \Theta \quad=$ $\left[\begin{array}{lllll}E_{2} L K(i) & 0 & \cdots & 0 & 0\end{array}\right]^{T}$.

By Lemma 2, there exists a scalar $\mu_{1}>0$ such that

$$
\left[\begin{array}{ccc}
-\sum_{j \in \Lambda_{k}^{i}} \pi_{i j} P(i) & * & * \\
\Lambda_{k}^{i}\left(\widetilde{A}_{0}+B L K(i) H(i)\right) & \widetilde{\Gamma}+\widetilde{\Xi}_{k}^{i} & * \\
E_{2} L K(i) H(i) & 0 & -\mu_{1}
\end{array}\right]<0,
$$

where

$$
\begin{aligned}
& \widetilde{\Xi}_{k}^{i}=-\operatorname{diag}\left\{P\left(k_{1}^{i}\right)^{-1} P\left(k_{2}^{i}\right)^{-1} \ldots P\left(k_{\mu}^{i}\right)^{-1}\right\}, \\
& \widetilde{\Gamma}=\left[\begin{array}{cccc}
\mu_{1} \widetilde{D} \widetilde{D}^{T} & * & * & * \\
\mu_{1} \widetilde{D} \widetilde{D}^{T} & \mu_{1} \widetilde{D} \widetilde{D}^{T} & * & * \\
\vdots & \vdots & \ddots & * \\
\mu_{1} \widetilde{D} \widetilde{D}^{T} & \mu_{1} \widetilde{D} \widetilde{D}^{T} & \cdots & \mu_{1} \widetilde{D} \widetilde{D}^{T}
\end{array}\right]
\end{aligned}
$$

Using Schur complement and Lemma 2 again, one can get

$$
\left[\begin{array}{cccc}
-\sum_{j \in \Lambda_{k}^{i}} \pi_{i j} P(i) & * & * & * \\
\Lambda_{k}^{i}\left(\widetilde{A}_{0}+\widetilde{B}_{0} L K(i) H(i)\right) & \Gamma+\widetilde{\Xi}_{k}^{i} & * & * \\
E_{2} L K(i) H(i) & 0 & -\mu_{1} I & * \\
\widetilde{E}_{1} & 0 & 0 & -\mu_{2} I
\end{array}\right]<0 .
$$


Similarly, from (13) one can obtain

$$
\left[\begin{array}{cccc}
-P(i) & * & * & * \\
\widetilde{A}_{0}+\widetilde{B}_{0} L K(i) H(i) & -P(j)+\left(\mu_{3}+\mu_{4}\right) \widetilde{D} \widetilde{D}^{T} & * & * \\
E_{2} L K(i) H(i) & 0 & -\mu_{3} I & * \\
\widetilde{E}_{1} & 0 & 0 & -\mu_{4} I
\end{array}\right]
$$$$
<0, \quad \forall j \in \Lambda_{u k}^{i}
$$

Performing a congruence transformation to (23) and (24) by $\operatorname{diag}\left\{P(i)^{-1}\right.$ I $\left.I I I\right\}$, setting $Q(i)=P(i)^{-1}$, one can obtain (25) and (26), respectively. One has

$$
\begin{aligned}
& {\left[\begin{array}{cccc}
-\sum_{j \in \Lambda_{k}^{i}} \pi_{i j} Q(i) & * & * & * \\
\Lambda_{k}^{i}\left(\widetilde{A}_{0}+\widetilde{B}_{0} L K(i) H(i)\right) Q(i) & \Gamma+\Xi_{k}^{i} & * & * \\
E_{2} L K(i) H(i) Q(i) & 0 & -\mu_{1} I & * \\
\widetilde{E}_{1} Q(i) & 0 & 0 & -\mu_{2} I
\end{array}\right]<0,} \\
& {\left[\begin{array}{cccc}
-Q(i) & * & * & * \\
\left(\widetilde{A}_{0}+\widetilde{B}_{0} L K(i) H(i)\right) Q(i) & -Q(j)+\left(\mu_{3}+\mu_{4}\right) \widetilde{D} \widetilde{D}^{T} & * & * \\
E_{2} L K(i) H(i) Q(i) & 0 & -\mu_{3} I & * \\
\widetilde{E}_{1} Q(i) & 0 & 0 & -\mu_{4} I
\end{array}\right]} \\
& <0, \quad \forall j \in \Lambda_{u k}^{i} .
\end{aligned}
$$

For the matrix $E(i)^{T}$ of full-column rank, there always exist two orthogonal matrices $U_{i} \in R^{n(1+d) \times n(1+d)}$ and $V_{i} \in R^{g \times g}$ such that

$$
E(i)^{T}=U_{i}^{T}\left[\begin{array}{c}
\Sigma_{i} \\
0
\end{array}\right] V_{i}^{T},
$$

where $\Sigma_{i}=\operatorname{diag}\left(\sigma_{1}, \sigma_{2}, \ldots \sigma_{g}\right), \sigma_{i}(i=1,2, \ldots, g)$ are nonzero singular values of $E(i)^{T}$. Assume that the matrix $Q(i)$ has the following structure:

$$
Q(i)=U_{i}^{T}\left[\begin{array}{cc}
Q_{i 1} & 0 \\
0 & Q_{i 2}
\end{array}\right] U_{i} ;
$$

according to Lemma 3, there exists matrix $X_{i} \in R^{n \times n}$ such that $F(i) E(i)^{T}=E(i)^{T} X_{i}$, setting

$$
X_{i} K(i)^{T}=Y(i)^{T}
$$

Since $F(i) E(i)^{T}=E(i)^{T} X_{i}$, one can get

$$
\left[\begin{array}{c}
\Sigma_{i} \\
0
\end{array}\right] V_{i}^{T}=U_{i}^{T}\left[\begin{array}{c}
\Sigma_{i} \\
0
\end{array}\right] V_{i}^{T} X_{i},
$$

which implies that

$$
X_{i}=V_{i} \Sigma_{i}^{-1} F_{i 1} \Sigma_{i} V_{i}^{T}
$$

Thus, (19) is obtained from (29) and (31), which completes the proof.

\section{Numerical Example}

In this section, a numerical example is given to show the validity and potential of our developed theoretical results. The dynamics are described as follows:

$$
\begin{gathered}
A_{0}=\left[\begin{array}{cc}
0.7 & -0.1 \\
0.1 & 0.3
\end{array}\right], \quad B_{0}=\left[\begin{array}{cc}
0.9 & 0.6 \\
0.7 & 1
\end{array}\right], \\
D=\left[\begin{array}{cc}
0.01 & 0 \\
0 & 0.02
\end{array}\right], \quad E_{1}=\left[\begin{array}{cc}
0.02 & 0 \\
0 & 0.03
\end{array}\right], \\
E_{2}=\left[\begin{array}{cc}
0.01 & 0 \\
0 & 0.03
\end{array}\right] .
\end{gathered}
$$

Assume the time-delay $\tau_{k}$ takes values from $\Lambda=\{1,2,3\}$ and the transition probabilities matrix is $\Pi=\left[\begin{array}{ccc}0.6 & ? & ? \\ ? & ? & 0 \\ ? & 0.8 & ?\end{array}\right]$. When the first actuator experiences a total failure, that is, the fault indicator matrix $L=\left[\begin{array}{ll}0 & 0 \\ 0 & 1\end{array}\right]$, the fault-tolerant and delay-dependent controller gain is solved from Theorem 5 as follows:

$$
\begin{gathered}
K_{1}=\left[\begin{array}{cc}
0.0109 & -0.002 \\
0 & 0
\end{array}\right], \quad K_{2}=\left[\begin{array}{cc}
0.0026 & -0.004 \\
0 & 0
\end{array}\right], \\
K_{3}=\left[\begin{array}{cc}
0.0100 & -0.0020 \\
0 & 0
\end{array}\right] .
\end{gathered}
$$

When the second actuator experiences a total failure while the first actuator works normally, that is, the fault indicator matrix $L=\left[\begin{array}{ll}1 & 0 \\ 0 & 0\end{array}\right]$, the controller gain is solved as follows:

$$
\begin{gathered}
K_{1}=\left[\begin{array}{cc}
0 & 0 \\
0.0091 & 0.0013
\end{array}\right], \quad K_{2}=\left[\begin{array}{cc}
0 & 0 \\
0.0022 & -0.0010
\end{array}\right], \\
K_{3}=\left[\begin{array}{cc}
0 & 0 \\
0.0080 & -0.0010
\end{array}\right] .
\end{gathered}
$$

When both actuators work normally, that is, the fault indicator matrix $L=\left[\begin{array}{ll}1 & 0 \\ 0 & 1\end{array}\right]$, the controller gain is solved as

$$
\begin{gathered}
K_{1}=\left[\begin{array}{cc}
0.0204 & -0.0098 \\
-0.0108 & 0.0108
\end{array}\right], \quad K_{2}=\left[\begin{array}{cc}
0.0048 & -0.0020 \\
-0.0024 & 0.0018
\end{array}\right], \\
K_{3}=\left[\begin{array}{cc}
0.0018 & -0.0007 \\
-0.0009 & -0.0005
\end{array}\right] .
\end{gathered}
$$

Zero-input responses of states $x_{1}, x_{2}$ are shown in Figures 2 and 3 when $x(0)=\left[\begin{array}{ll}-0.02 & 0.01\end{array}\right]^{T}$.

The curves of zero-input response states $x_{1}, x_{2}$ show that the NCS with partly unknown transition probabilities is stochastically stable against actuator possible fault. 


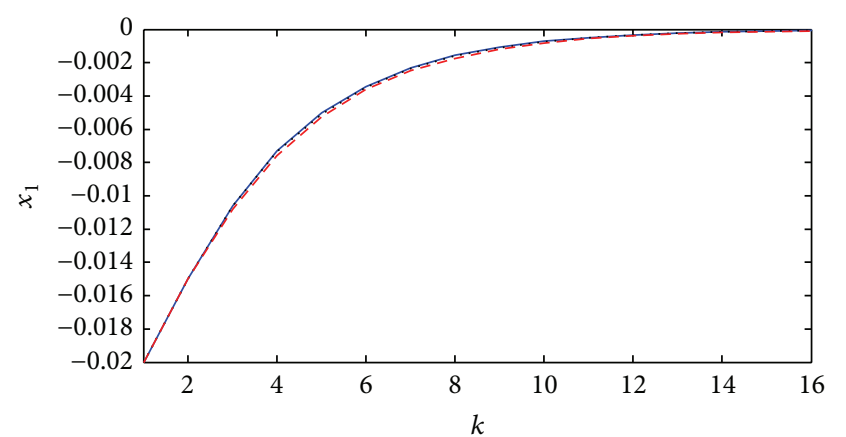

_... Actuator normal
_.. Actuator 1 failure
- - Actuator 2 failure

Figure 2: Zero-input response of $x_{1}$.

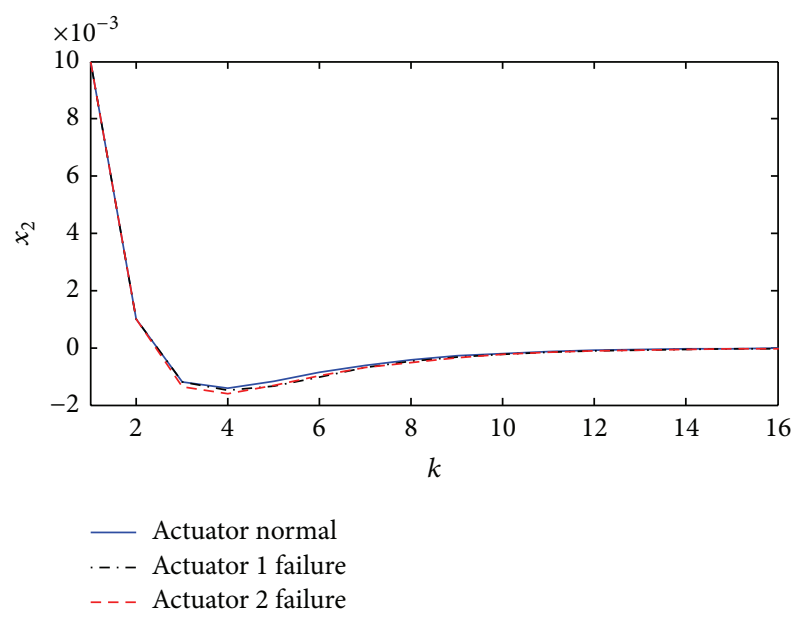

FIGURE 3: Zero-input response of $x_{2}$.

\section{Conclusions}

This paper is concerned with the problem of fault-tolerant control for uncertain discrete-time networked systems against actuator possible fault. The time-delay is modeled as a finite state Markov chain and the Markov chain's transition probabilities the information is limited. The closedloop system is established through the state augmentation technique and the state feedback controller is designed which guarantees the stability of the resulting closed-loop systems. It is shown that the controller design problem under consideration is solvable if a set of LMIs is feasible. Simulation results show that the closed-loop systems are stochastically stable against actuator fault.

\section{Conflict of Interests}

The authors declare that there is no conflict of interests regarding the publication of this paper.

\section{Acknowledgments}

This study is supported by the National Natural Science Foundation of China under Grant no. 61174029, the National Natural Science Foundation of China under Grant no. 61503136, the Zhejiang Provincial Natural Science Foundation of China under Grant no. LY12F03008, and the Huzhou Natural Science Foundation of China under Grant no. 2014YZ07.

\section{References}

[1] W. Zhang, M. S. Branicky, and S. M. Phillips, "Stability of networked control systems," IEEE Control Systems Magazine, vol. 21, no. 1, pp. 84-97, 2001.

[2] H. Ishii, " $H_{\infty}$ control with limited communication and message losses," Systems \& Control Letters, vol. 57, no. 4, pp. 322-331, 2008.

[3] J. Wu, H. R. Karimi, and P. Shi, "Network-based $H_{\infty}$ output feedback control for uncertain stochastic systems," Information Sciences, vol. 232, pp. 397-410, 2013.

[4] D. Wang, J. Wang, and W. Wang, " $H_{\infty}$ controller design of networked control systems with markov packet dropouts," IEEE Transactions on Systems, Man, and Cybernetics Part A: Systems, vol. 43, no. 3, pp. 689-697, 2013.

[5] L. X. Zhang, H. J. Gao, and O. Kaynak, "Network-induced constraints in networked control systems-a survey," IEEE Transactions on Industrial Informatics, vol. 9, no. 1, pp. 403-416, 2013.

[6] J. Zhang, Y. Xia, and P. Shi, "Design and stability analysis of networked predictive control systems," IEEE Transactions on Control Systems Technology, vol. 21, no. 4, pp. 1495-1501, 2013.

[7] X. Li and X. B. Wu, "Guaranteed cost fault-tolerant controller design of networked control systems under variable-period sampling," Information Technology Journal, vol. 8, no. 4, pp. 537543, 2009.

[8] Y. Zhang and J. Jiang, "Bibliographical review on reconfigurable fault-tolerant control systems," Annual Reviews in Control, vol. 32, no. 2, pp. 229-252, 2008.

[9] S. X. Ding, P. Zhang, C. I. Chihaia, W. Li, Y. Wang, and E. L. Ding, "Advanced design scheme for fault tolerant distributed networked control systems," in Proceedings of the 17th IFAC World Congress, pp. 13569-13574, Seoul, Republic of Korea, 2008.

[10] Z. H. Mao and B. Jiang, "Fault estimation and accommodation for networked control systems with transfer delay," Acta Automatica Sinica, vol. 33, no. 7, pp. 738-743, 2007.

[11] X. Qi, C. Zhang, and J. Gu, "Robust fault-tolerant control for uncertain networked control systems with state-delay and random data packet dropout," Journal of Control Science and Engineering, vol. 2012, Article ID 734758, 7 pages, 2012.

[12] D. M. Kong and H. J. Fang, "Stable fault-tolerance control for a class of networked control systems," Acta Automatica Sinica, vol. 31, no. 2, pp. 267-273, 2005.

[13] Z. H. Huo and Z. X. Zhang, "Research on fault-tolerant control for NCS with data packet dropout," in Proceedings of the 2nd International Symposium on Systems and Control in Aerospace and Astronautics (ISSCAA '08), pp. 1-4, IEEE, Shenzhen, China, December 2008.

[14] S. Klinkhieo, C. Kambhampati, and R. J. Patton, "Fault tolerant control in NCS medium access constraints," in Proceedings of the IEEE International Conference on Networking, Sensing and 
Control (ICNSC '07), pp. 416-423, IEEE, London, UK, April 2007.

[15] D. X. Xie, D. F. Zhang, G. Huang, and Z. H. Wang, "Robust fault-tolerant control for a class of uncertain networked control systems," Information and Control, vol. 39, no. 4, pp. 472-478, 2010.

[16] H. Gao, X. Meng, and T. Chen, "Stabilization of networked control systems with a new delay characterization," IEEE Transactions on Automatic Control, vol. 53, no. 9, pp. 2142-2148, 2008.

[17] Y. Wang, L. Xie, and C. E. de Souza, "Robust control of a class of uncertain nonlinear systems," Systems \& Control Letters, vol. 19, no. 2, pp. 139-149, 1992.

[18] F. Yang, Z. Wang, Y. S. Hung, and M. Gani, " $H_{\infty}$ control for networked systems with random communication delays," IEEE Transactions on Automatic Control, vol. 51, no. 3, pp. 511-518, 2006. 


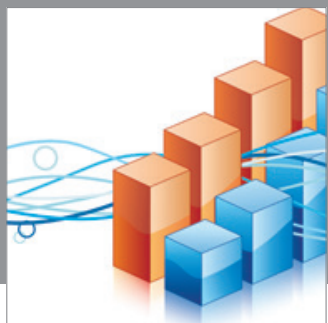

Advances in

Operations Research

mansans

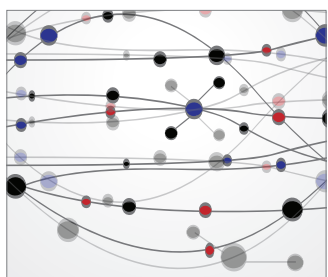

The Scientific World Journal
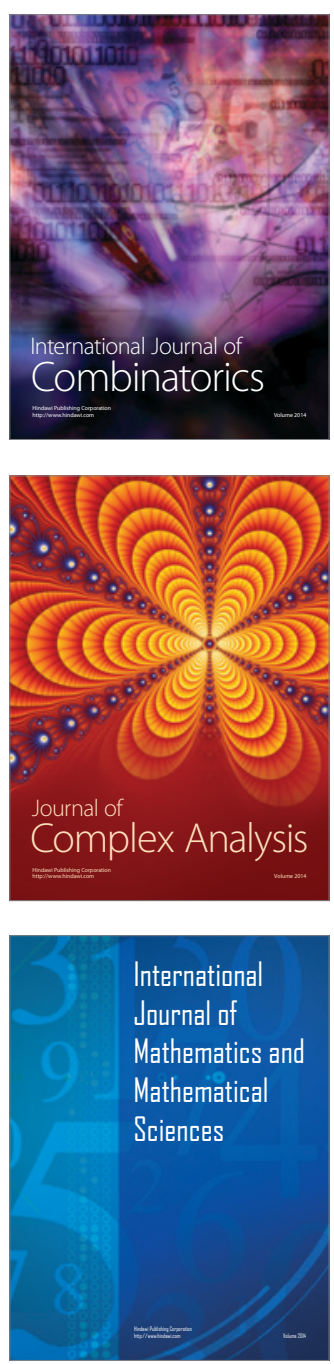
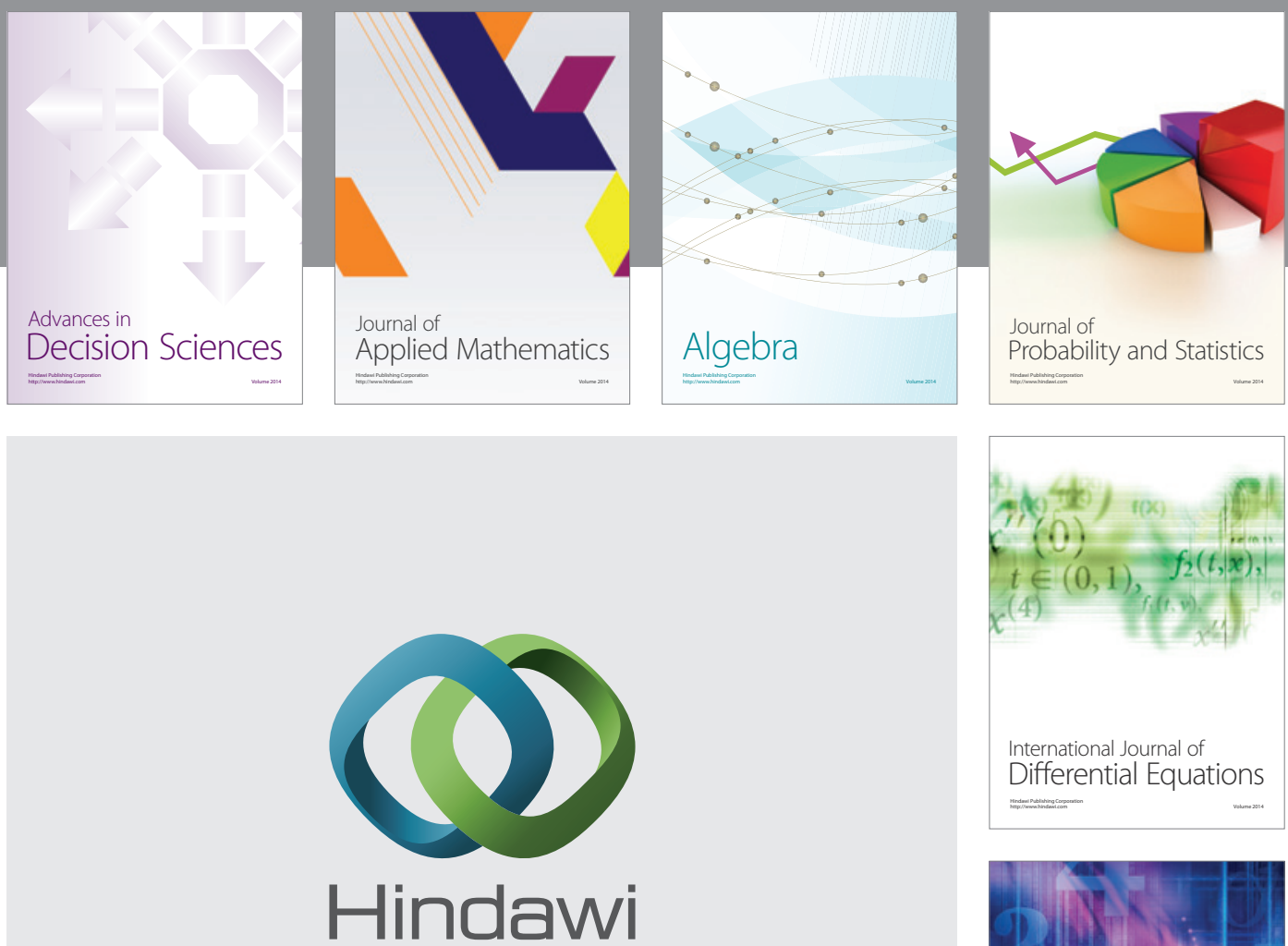

Submit your manuscripts at http://www.hindawi.com
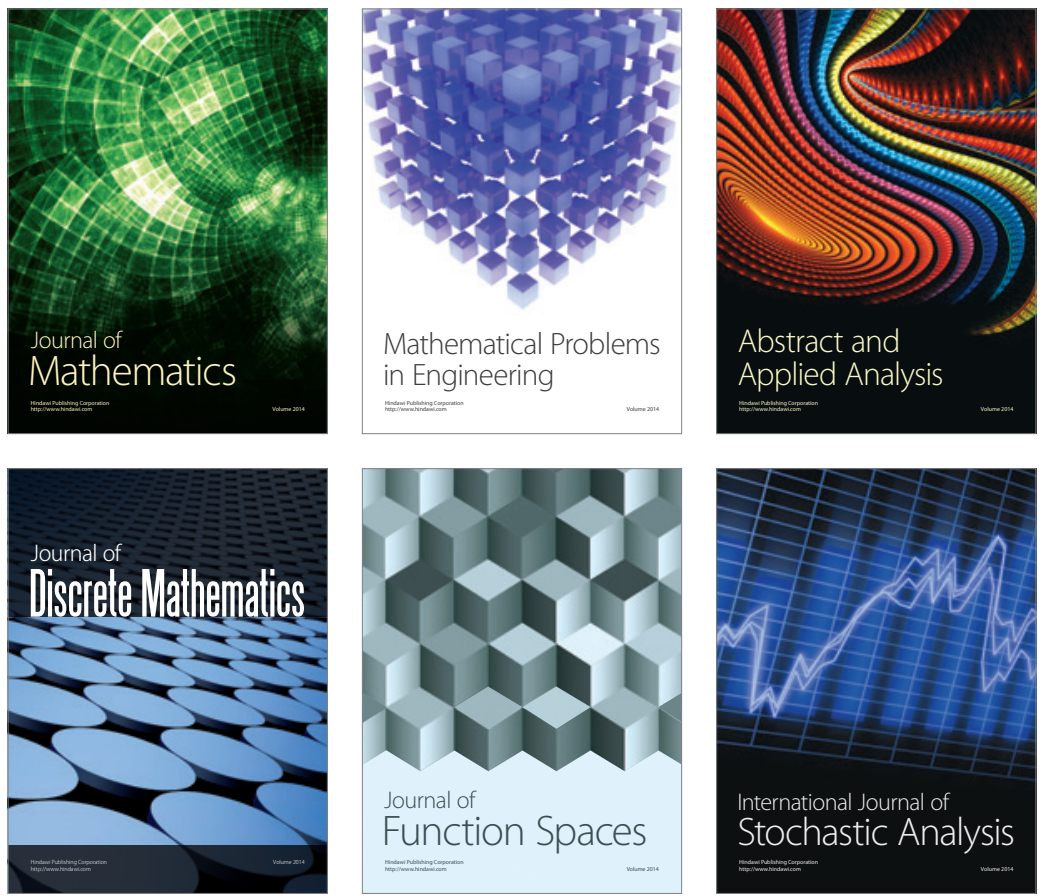

Journal of

Function Spaces

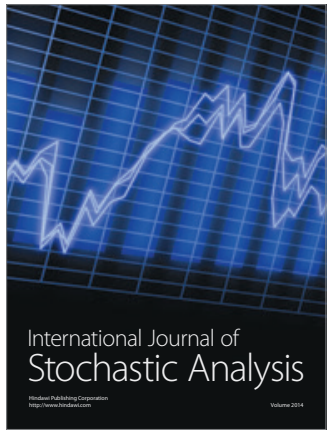

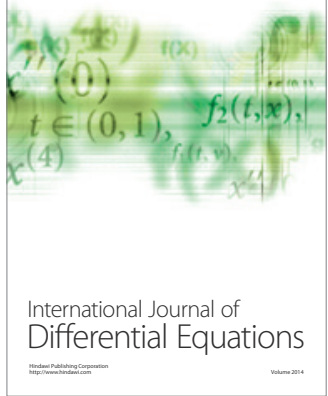
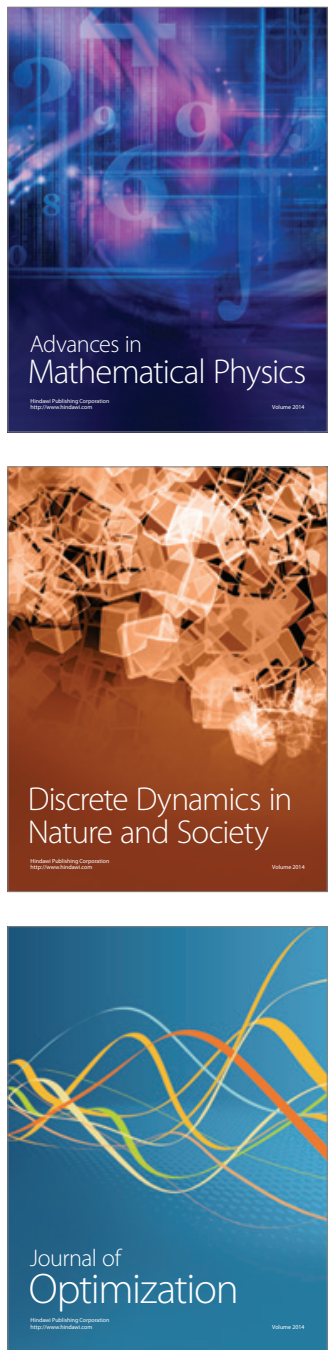